\title{
The ATLAS Magnets Test Facility at CERN
}

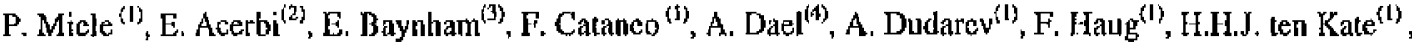 \\ G. Passardi ${ }^{(1)}$, E. Sbrissa ${ }^{(1)}$, P. Schilly ${ }^{(1)}$, A. Yamamoto ${ }^{(5)}$ \\ (1) CERN, Geneva, Switzerland; (2) INFN-LASA, Milano, Italy; ${ }^{(9)} \mathrm{RAL}_{r,}$ Chilton, UK; ${ }^{(4)}$ CEA, Saclay, France; ${ }^{(5)}$ KEK, \\ Tsukuba, Japan
}

\begin{abstract}
The magnet system for the ATLAS detector at CFHN consists of a Barrel Toroid (BT), two End-Cap Toroids (ECT) and a Central Solenotd (CS). The overall dimensions of the system are $20 \mathrm{~m}$ in diameter by $26 \mathrm{~m}$ in length. Before underground installation all coils will be tested on surface in a magnet test facility which is under construction. Moreover two model coils are tested as well as subsystems. In this paper the design and construction of the test facility is presented.
\end{abstract}

\section{INTRODUCTION}

The ATLAS Magnet System [1,2] comprises the superconducting magnets, the power supply system, the cryogenics system, the vacuum system and the control and safety system. The magnet system has overall dimension of 26 meters in length and 20 meters in diameler (see Fig. 1). The superconducting magnels comprise the Toroidal Field (TF) magnet system with the Barrel Toroid and the two End-Cap Toroids and the Central Solenoid. Each of the three toroids consists of eight coils connected in series, with flat pancake type of windings, assembled around the beam axis with an offset angle of $22.5^{\circ}$ between the BT and ECT systems, in order to optimize the bending power in the available space. $A$ model coil of the Barrel Toroid of roduced length, the $\mathrm{B} 0$ model coil, is under construction to qualify the manufacturing cycle and related techniques. The Central Solenoid is a single layer coil wound internally in a supporting cylinder and enclosed by the common cryostat of the Iriquid Argon Calorimeter, Aluminum stabilized $\mathrm{NbTi} / \mathrm{Cu}$ superconductor cooled at $4.5 \mathrm{~K}$ by liquid Helium foreed flow and glass fiberepoxy resin insulation are the main components of the coils. $A$ $21 \mathrm{kA}$ common TH power system and an $\mathrm{g} \mathrm{kA}$ CS power system with correspondent quench protection systems are implemented.

In order to verify the constriction concepts on $\mathrm{BO}$ and thereafter the correct operation of the other coils, the tests must represent the real configuration. The infrastrticture required to perform the tests comprises: a rather complex cryogenic plant with a $\mathrm{He}$ refrigerator, a pre-cooling unit, a cryostat with integrated immersed centrifugal pumps for liquid He flows above $80 \mathrm{~g} / \mathrm{s}$ to cool the magnet cold masses and a distribution system; the vacuum system with a pumping system able to provide $10^{-4}$ mbar; the electrical system for coil energizing with a $6 \mathrm{~V} / 2 \mathrm{AkA}$ power supply; appropriate quench protection units as well as systems for control and diagnostics.

Masugcript received Sept.,26, 1999

Paola Miele, e-mail: Paola,Miele@

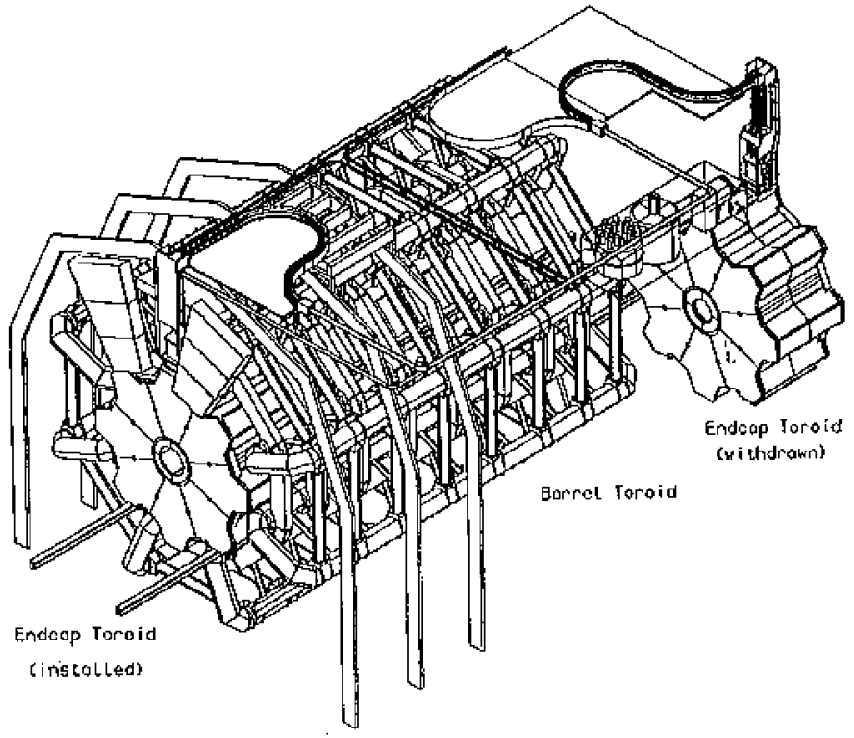

Fig. 1. The ATLAS Magnet System.

Proliminary test of the BO coil with a magnetic mirror to simulate the clectromagnotic load and the full set of instrumentation is planned by summer 2000. Due to the exceptional size of the $\mathbf{B} \boldsymbol{T}$ system, ench coil will be individually tested on the ground at nominal current with the magnetic mirror so as to reproduce the actual load, before lowering them down in the cavern. Each of the two ECT magnets and the CS magnet will be fully assembled and tested on the surface, before the installation as complete systems in the cavern.

\section{THE BOO AND B0 MODEL COIS}

The first coil to be tested is the B00 model coil planned for beginning of the year 2000 . The main purpose of the B00 model coil is to test all types of ATLAS superconductors in the operating conditions to study ramp losses, stability and quench propagation. Moreover it serves to commission the test station.

The $\mathrm{B} 00$ includes:

- Double pancake winding made with BT conductor;

- Double pancake winding made with ECT conductor;

- Single pancake winding marle with CS conductor.

An Al coil casing with glued Al alloy tubes for cooling of the cold masses by liquid He is implomented.

The next coil, the BO model coil, is a test-model of the BT coils with the same design and construction procedures, so as 


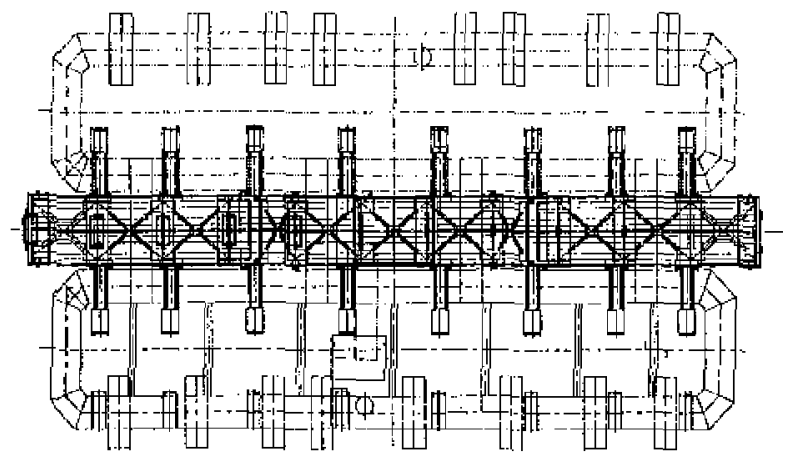

Fig, 2. The 2 BT test bencles: top wiew. The magetic miror with its supporl structure is visible in the middle.

to validate the numufacturing feasibility of the coils. The width and cross scetion of the BO coil ate the same as for the BT coils, while a reduced length of $9 \mathrm{~m}$ was chosen to reduce the cost and the test requirements.

"To have as many information as possible many diagnostics and instrumentation are implemented in the BO coil, such as temperature sensors, voltage taps, pick-up coils, quench heators, point heaters, strain gauges, hall probes in the joints, superconducting quench detectors, Rogowski coils, acoustic probes and positioning sensors. Morcover diagnostics are required ajso in the exterual services, namely He flow sensors on current leads, current leads theaters, DCCT, power supply readings, cryogenics readings, vaeuum readings and water flow readings.

The test of $\mathrm{B} 0$ witl the magnetic mirror is performed only in the horizontal position. In fact in the inclined positions, simulating the real orientation of the BT magnets, only a $5 \%$ contribution to cold-to-warm support forces is given by gravity. For testing the cold mass supports and the internal oryogenics a turnover test withoul magnetic mirrors is sutficient to simulate the loads. This is also required as an assembling and handling exercise.

A detailed test plan is being prepared in order to verify and control the following mait issues:

- Thermal cycling between $300 \mathrm{~K}$ and $4.5 \mathrm{~K}$ and measurements of the related thermal gradients and electrical properties;

- Current cycling up to the current of $24 \mathrm{kA}$ with operation at various current levels and verification of the mechanical

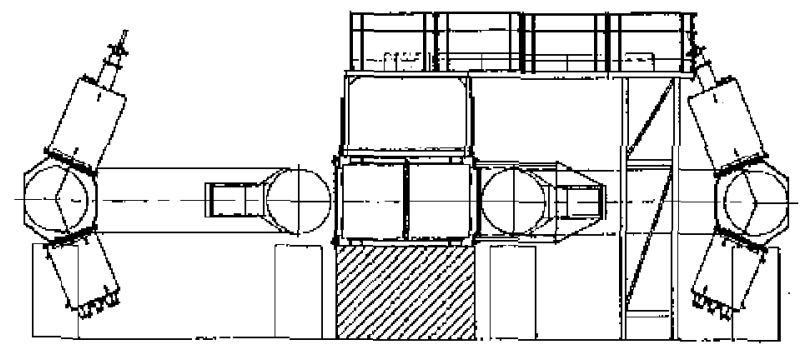

Fig. 3. The two BT test bencles: lateral viow. stresses in the entire structure and of the salety of the fast and slow discharge procedures;

- In each of the preceding phases, verification of the ground insulation, measurement of the inductance and of the potential in the pancakes for the detection of any internal changes and check of any mechanical disturbances by acoustic sensors;

- Magnetic field measurements

The test of $\mathrm{BO}$ is of the utmost importance because it allows checking the proper functioning of both the clyogenic, electrical and mechanical sensors and the control, safety and data acquisition systoms foreseen for the toroids.

\section{I11. 'THF ATLAS MAGNET SYSTEM}

\section{A. Barrel Toroid}

Due to tho exceptional size of the BT magnet system, it is not feasible to assemble and test the integrated system on surface, before installation in the underground cavern. Therefore each coil after integration will be individually tested on surface and then lowered down into the cavern for the final assembly of the toroid. "Two temporary turrets ate installed for the test campaign on each coil, a cold turtet which connects to the cryogenic transfer lines through flexible lines and a warm turter which connects to the bus bars via $24 \mathrm{kA}$ current leads. Feeding of the He is preferably done from the bottom, so as to facilitate the distribution in the internal cooling tubes. Tho magnetic mirror is installed between two coils and held in position by a dedicated support structure, so as to simulate the actual elcctromagnetic lension load 1280 tons experienced in the real conliguration (sec Fig. 2 and Fig. 3). The time schedule foresees 2.5 yoars for the test of eight BT coils starting in spring 2001, with 5 or 6 months time allocated for each magnet. This requires work to be done in parallel in the two test benches.

\section{B. End-Cap Toroids}

The ECT (Fig. 4) will be fully tested before transfer to the ATLAS cavern. A tost outside Hall 180 is foresecn using the same test facility as for BT coils. The cold masses and vacuum vessels are fully assembled and tesed at $80 \mathrm{~K}$ in Hall 191 . Then they are transferred to the test station position. The cryogenics transfer lines and the bus bar lines will be routed outside Hall 180 probably from the top and attached to the special service turret of the ECT,

The main diffusion pumps and all the control, monitoring and safety cabling are comnected to the service turret. The tesi time allocated for the End-Cap Toroids is 1.5 years starting form the beginning of the yeat 2003.

\section{Central Solenoid}

The CS and Liquid Argon Calorimeter are assembled in a common eryostat. A full excitation test of the coil is done at the coil manufacturer side in a temperary cryostat but already assembled on its final inner cylinder. The power supply, the 


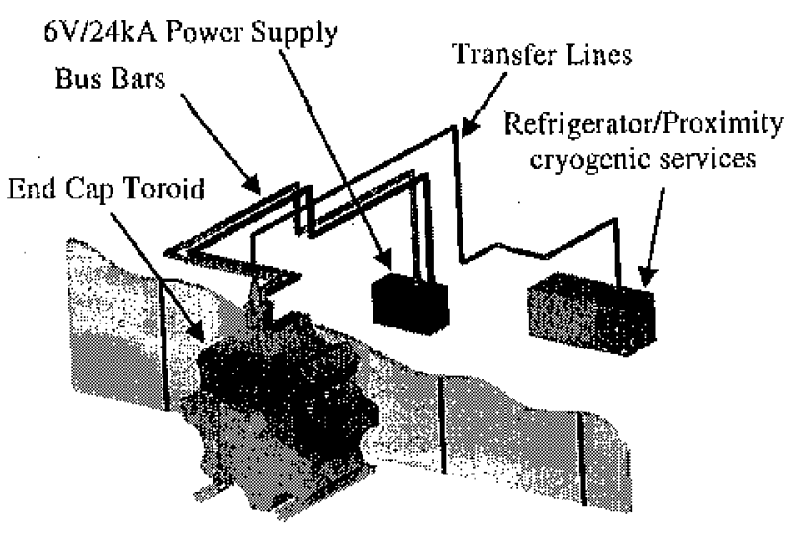

Fig. 4. ECT test station.

control dewar and MCS/MSS systems are as closely as possible to the linat ones. Thereafter it is sent to CERN and tested fully integrated with the Liquid Argon Calorimeter in Hall 180. A complete separate infrastructure is required for the test. The excitation test at "Toshiba", Japan is planned by the summer of the year 2000, while the test at CERN starts at the end of 2002 .

\section{TEST STATION}

The assembly and testing of all the coils require an important infrastructure in terms of a hatl space, hall height, lifting capacitics and services, The West Arca at CERN meets such requirements (see Fig. 5), Building 180 has a space available for the ATLAS magnets of $7300 \mathrm{~m}^{2}$. This space just meets the requitements for the tes: and the assembly of the BT and CS magnets, while the ECT magnets will be assembled in Hall 191 and tested outside Hall 180. Two cranes with a capacity of 40 and 60 tons cover the whole arca of Hall 180 . The capacity of $100 \mathrm{~T}$ is necessary to lift 1 BT magnet with support structures and lifting tools.

The BT coils are the most demanding in terms of space.

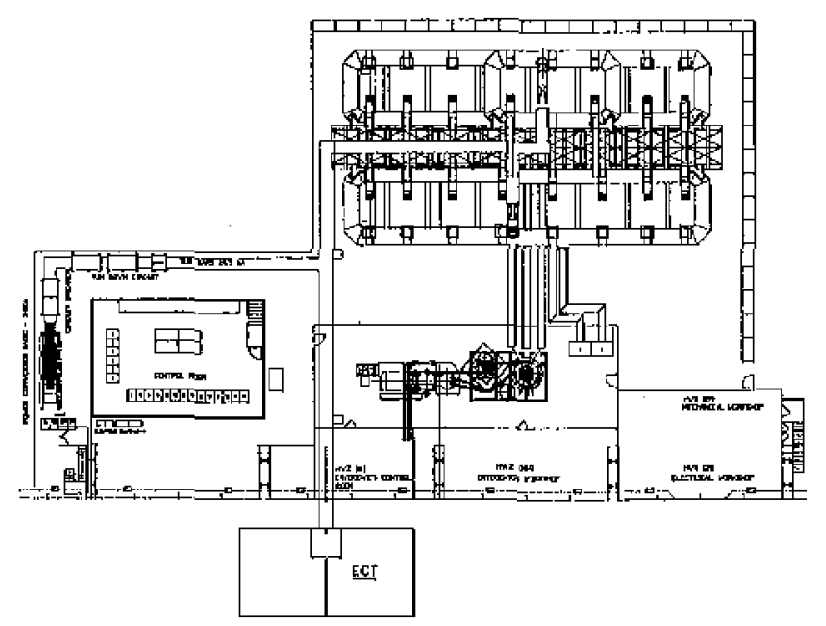

Fig. 5. The test stalion in Hall 180 at CERN
After manulacturing of the coil components, they will be delivered to CERN ready for integration, which consists of the installation of the cold mass in the vacuum vessel with all the related components. The BT test station comprises two test benches; the cryogenic and power supply systems with related control rooms and workshops; the group of partips of the vaculsm system and the magnet control room. First BOO will be delivered to CERN iully assembled and its test is planed in the bottom test bench (see Fig.5). Then the B0 coil will arrive and will be tested in the top position. The same test cquipment in terms of external services will he used for the test of the ECT magnets fully assembled, while the CS coil requires a separate system. Threc transfer lines distribute gaseots and liquid helium from the distribution valve box to the individual coils with flexible lines at the BT cryogenic supply terret and the ECT service turret. Bus bar lines distribute the current from the power supply to the magnets with the $24 \mathrm{kA}$ current leads that torminate at the $\mathrm{BT}$ current lead turrel and the ECT service turret.

A full functional test of each coil will be carried out in the test station, which comprises:

- Warm check of the electrical and thermal properties and interlocks;

- Pump down of the vacuum vessel and related leak test;

- Cool down between 300 and $100 \mathrm{~K}$ in 20 days and between 100 and $4.5 \mathrm{~K}$ in 10 days with related cryogenics tests;

- Coil tests with current ramps up to $21 \mathrm{k} \Lambda$ and quench test; - Warming up.

\section{CRYOGRNICS SYSTEM}

'The cryogenies system [3] is based on immersed centrifuga] pumps to circulate two phase He flow of nominally $80 \mathrm{~g} / \mathrm{s}$ through the cooling pipes of the magnet cold masses. For the thormal shields $60 \mathrm{~K}$ Ho gas is supplied. The current loads require $3 \mathrm{~g} / \mathrm{s}$ liquid flow. This cryogenic system provides al] the necessary functions including the cool down of the magnets from ambient to baseline temperature and flexiblo operational conditions.

It comprises the refrigerator cold box $1.2 \mathrm{~kW}$ at $4.5 \mathrm{~K}$ and related compressor; the pump cryostat containing two immersed centrifugal pumps in their anti-cryostats, the distribution valve box, the liquid nitrogen pre-cooling unit and related comprossor, three transfer lines fecding the test benches, the instrumentation and the process controls. The equipment will be installed and commissioning will be completed by the end of the year 1999 .

The pump cryostat and its instrumentation were tested and the principle of immersed centrifugal He pumps has been validated. Preliminary performance characteristics of the pump were obtained and the technical specifications were confirmed. The use of immersed pumps with its specilic design gives an extra safety measure in the case of failure of the refrigerator allowing for 1 hour of autonony. This covers twice the time required for a slow discharge of the BT coil. Commissioning of the refigerator and related compressor was successfully accomplished. A combined test of the cryostat and 


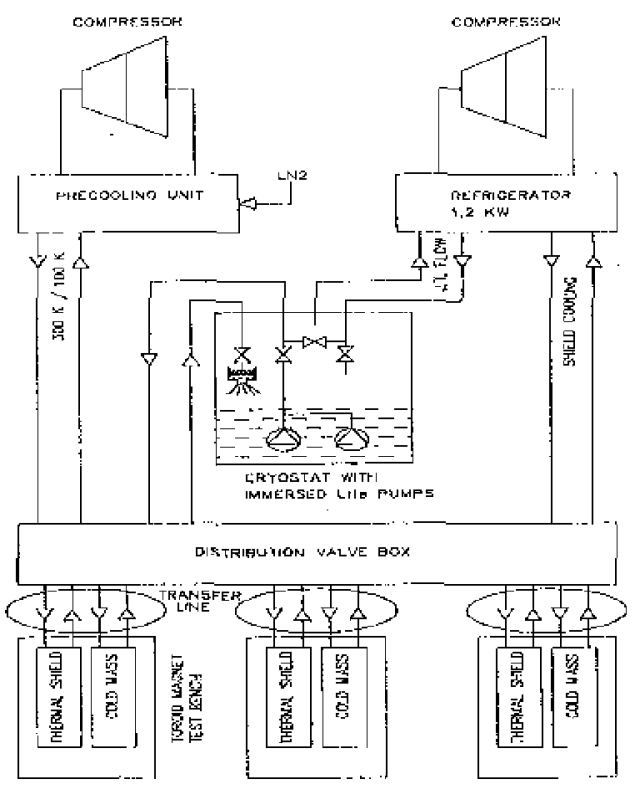

Fig. 6. Flow Scheme of the Cryogenic System.

tefigerator to verify the functioning of the different components, namely the flow meter, the phase separator, the quality meter was carried out. In this context tho development of a new quality meter, the void fraction meter and the construction and testing of a Venturi flow meter at $4.5 \mathrm{~K}$ was carticd out. Moreover the common control system was tested through simulation of the coil thermal losses by heaters, the safety presstre control valve for the lailure, mode and the liquid He consumption for the magnet dischatge scenario were tested. The simplificd flow scheme of the cryogenics system is shown in Fig. 6.

\section{POWER SUPPLY SYSTEM}

A 6 V/24kA power supply of the switch mode type to charge the magnets and a $45 \mathrm{~V} / 450 \mathrm{~A}$ auxiliary power supply to finally warm up the coils are required for the test. The implementation of the power supply in switch mode has the main advantage of reducing the volume of the power supply and introducing mordularity and redundancy. The system comprises a power converter and DCCTs, circuit breaker, dump resistor and diodes unit, bus bars and flexible connections, current leads, coil satcllites, 230/400 VAC main distribution and control room for the data acquisition and analysis. For safety reasons a 230 VAC uninterruptible power supplies are used to supply essential parts of the power system which must guarantec the safety of the magnet. Installation and commissioning of all these components are completed by the beginning of 2000 .

\section{MAGNET CONTROL ANN SAEETY SYSTEMS}

For testing of the coils, a prototype of the control and safety interlock system of the final system in the underground cavern will be used. For reasons of uniformity, ease of operation and maintenance, the systems are built from similar modules in a layered architecture. The main functions of MSS are the magnet protection in a stand-alone mode with fast and slow dump request treatment and the personnel safety. Auxitiary functions are the data provision for diagnostics and the alarm system. Uninterruptible power supplies are required for powering the system so as to guarante the functionality of MSS during at least the time of a slow discharge. Dual redundancy is implemented: The main functions of MCS are the process control for onsuring the operating sequences of the magnets and the monitoring and control of all the functional parameters.

The system provides control over the following:

- Magnet parameters;

- Internal and proximity cryogenics;

- Power supply and electrical system, bus bars and current leads;

- Vacuum system.

\section{CONCLUSIONS}

A preliminary test of B00 model coil, which represents the different conductors of the $\Lambda \mathrm{TJ}$.AS magnet system, will verily the superconductor performance and will commission the test station. Thereafter the $\mathrm{B} 0$ model coil will be tested and the main functional aspects of the toroid construction will be verified. Moreover testitig of the ECT and the CS is a crucial step to demonstrate the reliability of such a complex magnet. system, which for its exceptional size and techtological processes represents a challenge in both physics and cngineering research and devolopment.

\section{ACKNOWI.HDGEMENTS}

Thanks are due to J.M. Maugain, E. Towndrow, A. Moral, $\mathrm{X}$. Sun for their contributions to this paper.

\section{REFERENCES}

[I] ATLAS Magnet System Technical Design keport - 4 Volnmes, CERN doeumeut CERN/IHHC/97 $18,19,20,21,1997$.

[2] If ten Kate, 'Superconducting Magnet System for the ATLAS Detcetor at CERN, IEEE 7trans. on Appl. Supercond., Vol. 9, N2, p.161, 1999

[3] F Haug at al, "The CERN Cryogenic Test Fncility for the ATLAS Barrel Toroid Magnets', these Procedings. 